\title{
Terahertz optics taking off
}

\author{
Advances in terahertz sources and measurement techniques are opening up new opportunities for \\ fundamental material research in the terahertz region.
}

Terahertz $(\mathrm{THz})$ radiation, widely defined as electromagnetic radiation in the frequency range $0.3-3 \mathrm{THz}$, is attracting considerable interest owing to potential applications in imaging and spectroscopy for medical diagnostics and biology, highbandwidth communication, security and defence, and non-destructive evaluation.

$\mathrm{THz}$ optics has entered a new phase of research in recent years. The performance of $\mathrm{THz}$ sources has been significantly enhanced so that they now have considerably higher output powers, narrower pulses (in both the time and frequency domains) and wider frequency ranges. Furthermore, the increase in their degrees of freedom makes $\mathrm{THz}$ sources just as convenient as visible or infrared sources for pump-probe measurements. Finally, THz radiation can be used to obtain important physical data that is not accessible using X-ray or infrared radiation. Several measurement techniques based on $\mathrm{THz}$ radiation are now employed for fundamental material research.

For example, $\mathrm{THz}$ radiation is used to manipulate spin and the superconductivity of materials.

Clearly, the interest in $\mathrm{THz}$ science extends beyond the development of light sources and measurement techniques to fundamental material research. This issue of Nature Photonics features a special focus on $\mathrm{THz}$ optics with three Reviews, a Commentary, and an Interview, which showcase the exciting state of current research in this area.

In the Interview on page 670 , $\mathrm{Xi}$-Cheng Zhang recalls the early development of $\mathrm{THz}$ science in the late 1980s and early 1990s. He identifies two milestones in the development of $\mathrm{THz}$ sources - the generation of $\mathrm{THz}$ pulses from short laser pulses and the development of the $\mathrm{THz}$ quantum cascade laser. He envisages that the next breakthrough in the field could be the development of high-intensity $\mathrm{THz}$ sources. He says that non-destructive $\mathrm{THz}$ imaging is highly promising for meeting the current demands in the areas of security and quality control. He also considers that combining different technologies, such as electronics and plasmonics, is important for realizing novel $\mathrm{THz}$ systems that offer

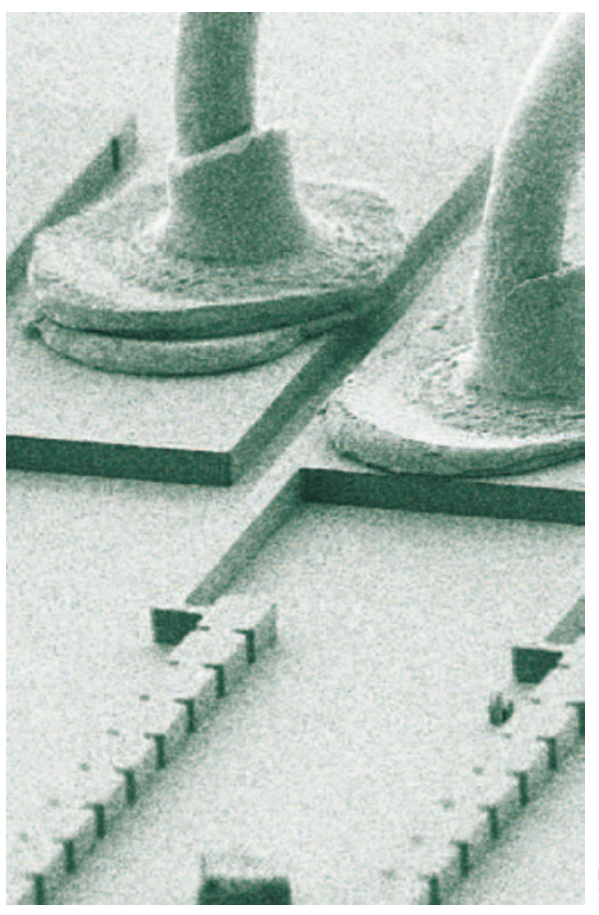

orders of magnitude improvements in performance at a fraction of the cost of existing systems.

In their Review on page 680, Tobias Kampfrath and co-authors describe how intense $\mathrm{THz}$ transients interact with molecules in condensed matter and gases, and how they can be used to manipulate spin, electrons, lattice vibrations, molecular orientation and rotation. In addition to resonant manipulation, the Review also considers nonresonant manipulation such as field ionization of impurities, high-field electron transport and impact ionization.

Coincidentally, two of the research papers in this issue also fall in the area of terahertz transients. On page 720, Carlo Vicario and co-workers report off-resonant magnetization dynamics in a cobalt thin film. The strong phase locking between an intense single-cycle $\mathrm{THz}$ wave transient and the magnetization permits the magnetization vector of a $\mathrm{THz}$ magnetic field to be directly controlled on a femtosecond timescale. This finding promises to open up new avenues for realizing ultrafast data recording in magnetic media.
On page 724, Masaaki Sato and co-workers report a new experimental technique for polarization shaping of terahertz pulses to arbitrarily control the chirality, phase, pulse duration and frequency of circularly polarized few-cycle $\mathrm{THz}$ pulses. This technique has numerous potential applications in areas such as nonlinear optics, coherent control and coherent multidimensional spectroscopy for which the polarization degree of freedom is important.

$\mathrm{THz}$ quantum cascade lasers have evolved dramatically since they were first developed. On page 691, Carlo Sirtori and co-authors review $\mathrm{THz}$ engineering using quantum cascade lasers and highlight methods that have been used to control their spectral and beam properties. On the other hand, on page 702, Ulrich Welp and co-authors provide an overview of the state-of-the-art research on superconducting $\mathrm{THz}$ emitters based on $\mathrm{Bi}_{2} \mathrm{Sr}_{2} \mathrm{CaCu}_{2} \mathrm{O}_{8+\delta}$. The development of $\bar{\Sigma}$ superconducting sources of $\mathrm{THz}$ - and sub-THz radiation is of technological importance, because this frequency range remains difficult to cover with compact solid-state sources.

The Commentary by Daniel Mittleman on page 666 notes the catch-22 situation that used to exist in $\mathrm{THz}$ research - there was no strong incentive to develop new technologies without first identifying a 'killer application', but there was little motivation to explore applications without establishing a viable technology base. He points out that the development of innovative tools and techniques is vital for improving research capabilities and opening up new research directions in the terahertz regime. He cites the recent progress in $\mathrm{THz}$ sources and plasmonics as two examples of exciting advances that contribute to the vibrancy of $\mathrm{THz}$ research and technology.

$\mathrm{THz}$ radiation shares some characteristics with both visible light and microwaves. This intermediate nature of $\mathrm{THz}$ radiation can make it useful for a wide range of applications and give it an advantage over electromagnetic radiation in other frequency regimes. This focus issue provides an overview of the importance and applications of $\mathrm{THz}$ radiation. 